\title{
Histologic Studies of the Bypassed Stomach after Roux-en-Y Gastric Bypass in a Porcine Model
}

\author{
Paolo Gentileschi, MD; Michel Gagner, MD, FACS, FRCSC; Luca Milone, \\ MD; Subhash Kini, MD; Shoji Fukuyama, MD
}

Department of Surgery, New York Presbyterian Hospital, Weill College of Medicine of Cornell University, and Mount Sinai Hospital, New York, NY, USA

Background: Laparoscopic Roux-en-Y gastric bypass (LRYGBP) is highly effective for morbid obesity. However, the long-term effects in the bypassed segments are unknown. The aim of this study is to evaluate gastrin and histologic changes in bypassed segments after LRYGBP.

Methods: 10 50-kg pigs were subjected to LRYGBP. Preoperative weight and serum gastrin were compared with similar measures at 6 months postoperatively, when the pigs were euthanized. At necropsy, full-thickness gastric, duodenal, and jejunal biopsies were performed. Normal biopsies were obtained from a control group of 10 pigs.

Results: 1 pig died at 3 months postoperatively because of an intestinal intussusception. In the remaining 9 pigs, weight increased after surgery from $52 \pm 2.2 \mathrm{~kg}$ to $55 \pm 1.9 \mathrm{~kg}$. Serum gastrin was unchanged after surgery (mean $68.2 \mathrm{vs} 68.3 \mathrm{pg} / \mathrm{mL}$ at 3 months and $61.7 \mathrm{pg} / \mathrm{mL}$ at 6 months). Histology showed no abnormalities from sections in all control pigs, and in 7 of the LRYGBP pigs as well. 1 LRYGBP pig was found to have hyperplastic duodenal glands, jejunal mucosa with mild chronic inflammation, and gastric mucosa with focal erosive gastritis. 1 LRYGBP pig had jejunal sections showing Peyer's patches.

Conclusion: LRYGBP is not associated with gastrin changes and major histologic changes in the bypassed segments, at 6 months postoperatively in the porcine model.

Key words: Laparoscopic, Roux-en-Y, gastric bypass, bypassed stomach, gastric histology, gastrin

Reprint requests to: Michel Gagner, MD, FACS, New York Presbyterian Hospital, Weill College of Medicine of Cornell University, Department of Surgery, 525 East 68th Street, Box 294, New York, NY 10021, USA. Fax: (212) 746-5236;

e-mail: mig2016@med.cornell.edu

\section{Introduction}

Morbid obesity is present when body mass index (BMI) is $\geq 40 \mathrm{~kg} / \mathrm{m}^{2}$ or $>35 \mathrm{~kg} / \mathrm{m}^{2}$ with concomitant obesity-related morbidity. Surgery is the only effective long-term treatment for morbid obesity. ${ }^{1}$ A recent meta-analysis confirmed that bariatric surgery is the appropriate therapy for morbidly obese patients in whom non-surgical treatment options have failed. ${ }^{2}$

The Roux-en-Y gastric bypass, either performed through an open (RYGBP) or laparoscopic approach (LRYGBP), has become the procedure of choice for morbid obesity in the USA. ${ }^{3}$ Most studies report a weight loss of $60 \%$ to $70 \%$ of excess body weight, with long-term weight loss extending to 10 years and longer. ${ }^{1,4}$

In creating a tiny gastric pouch, a major portion of the stomach is bypassed. Long-term effects in the bypassed segment remain unknown. Some authors have described carcinoma developing in the excluded stomach, but the real problem remains unknown. ${ }^{5-8}$

Gastrin is secreted from the G-cells of the stomach and the duodenum; these are stimulated by parasympathetic activity. Gastrin's role is to promote gastric distension and inhibit acid secretion from the parietal cells. Other factors that contribute to the acid secretion are acetylcholine release as a transmitter by parasympathetic activity and locallyreleased histamine.

The aim of this study is to evaluate serum gastrin levels and histologic changes in the bypassed segments after LRYGBP, using the porcine model. 


\section{Methods}

Institutional approval by the animal ethics committee of the Mount Sinai School of Medicine was obtained before commencing the research project. Ten $50-\mathrm{kg}$ pigs underwent LRYGBP. Pigs were selected because of their anatomical similarity to humans and their size, which make them highly suitable for the proposed bariatric surgery study. They were housed in the animal-care facility for 7 days before surgery for acclimatization. Preoperative baseline evaluation of the gastrin level was performed. Weights of the pigs were recorded before surgery.

On the morning of surgery, each pig received intravenous fluid, was sedated with ketamine, received pentobarbital intravenously for induction of anesthesia, and had an endotracheal tube positioned. The animal was then placed on a ventilator and received isoflurane for anesthesia. LRYGBP was performed according to the technique described by Gagner. ${ }^{9}$ All animals were allowed to recover from anesthesia. Bupivacaine and butorphanol were administrated intramuscularly twice a day for 2 days after the operation. The animals were fed pig chow for 6 months.

Each animal underwent a blood test for gastrin evaluation at 3 and 6 months, and weight was recorded once a month. After 6 months, the animals were euthanized, and necropsy was performed. The stomach was removed and sent to the pathologist for macro- and microscopic examination.

A control group of 10 pigs that did not undergo the gastric bypass operation was compared. After 6 months, they were euthanized and their stomachs sent to the pathologist for examination. No blood sample was taken in the control group because the authors assumed gastrin levels to be normal as opposed to the post-surgical group.

\section{Results}

Nine of the 10 pigs survived for the duration of the study. After 3 months, one animal had hematemesis and was euthanized; the necropsy showed a jejunojejunal intussusception of approximately $30 \mathrm{~cm}$.

The animals' weights increased $5.7 \%$, from $52 \pm 2 \mathrm{~kg}$ before surgery to $55 \pm 2 \mathrm{~kg} 6$ months after surgery. The mean gastrin value before surgery was $68.2 \mathrm{pg} / \mathrm{mL}$; the results obtained after 3 and 6 months were 68.3 and $61.7 \mathrm{pg} / \mathrm{mL}$, respectively. Table 1 shows the gastrin values of the 10 animals during the 6-month period.

Histology showed no abnormalities in the sections from 7 pigs and in all animals of the control group. One LRYGBP pig was determined to have hyperplastic duodenal glands, jejunal mucosa with mild chronic inflammation of tips of villi and numerous eosinophils, and gastric mucosa with focal erosive gastritis and areas of regenerative epithelium without dysplasia (Figures 1 and 2). One LRYGBP pig had jejunal sections with isolated mucosal lymphoid follicles (Peyer's patches) (Figure 3).

Most jejunal sections disclosed Peyer's patches determined to be of normal size. No evidence of metaplastic changes, atrophic gastritis, or dysplasia was noted. The two-way randomized block analysis of variance (two-way ANOVA), used to compare the level of gastric change, showed no significant differences across time in all the animals $(P=0.3)$ (Figure 4).

\section{Discussion}

The bypassed stomach after gastric bypass is difficult to study because of the lack of continuity with the tiny proximal gastric segment that makes it a blind excluded viscus. At the beginning of RYGBP history, the biliopancreatic (afferent) limb length was approximately $50 \mathrm{~cm}$ and the Roux limb length was also 50 $\mathrm{cm}$ on average. Thus, the distance between the mouth and gastric remnant was $80-100 \mathrm{~cm}$, allowing retro-

Table 1. Gastric values $(\mathrm{pg} / \mathrm{mL})$ in the 10 pigs

\begin{tabular}{lcc}
\hline Pre-op & $\mathbf{3}$ months & $\mathbf{6}$ months \\
\hline 48 & 62 & 42 \\
82 & 88 & 66 \\
50 & 62 & 46 \\
38 & 61 & 40 \\
62 & 48 & 60 \\
56 & & \\
94 & 86 & 84 \\
90 & 76 & 88 \\
120 & 78 & 90 \\
42 & 54 & 40
\end{tabular}




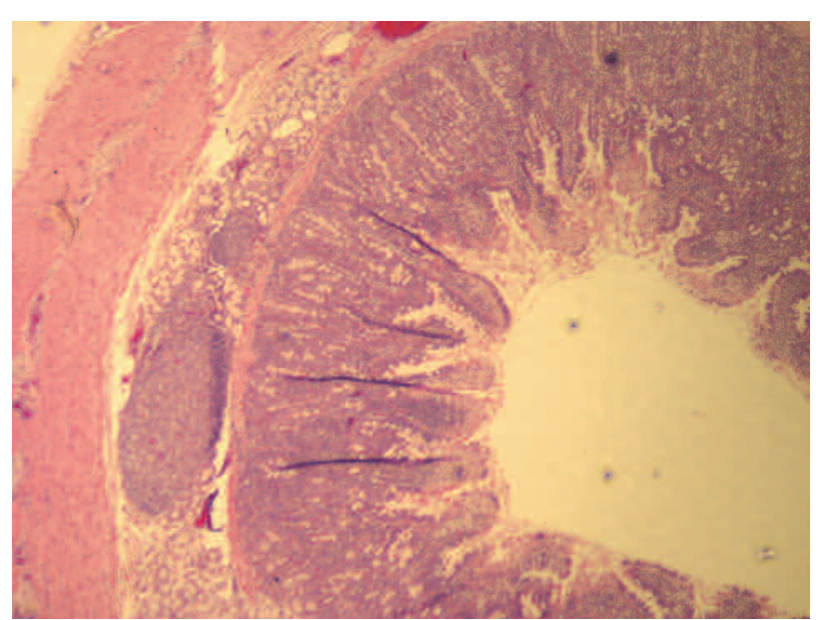

Figure 1. Hyperplastic duodenal glands.

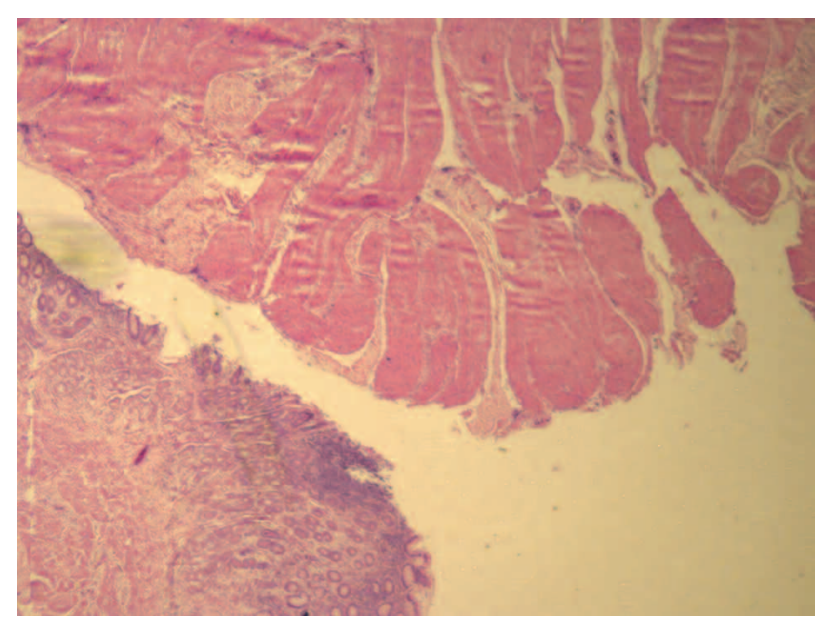

Figure 2. Erosive gastritis.

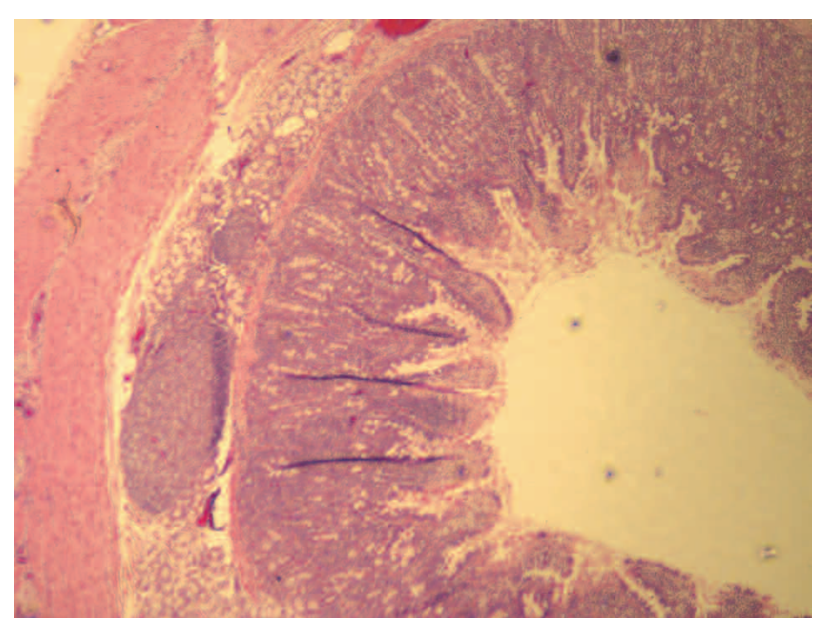

Figure 3. Duodenum with lymphoid follicles.

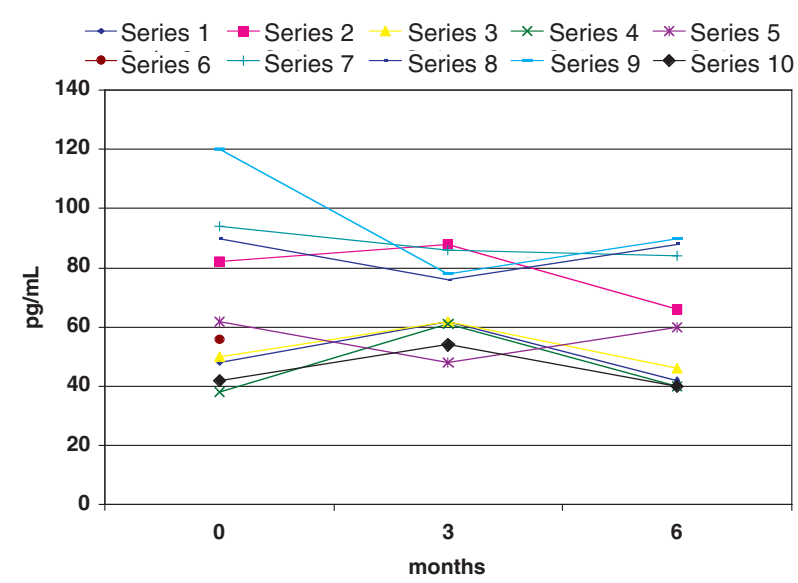

Figure 4. Changes in gastrin levels.

grade visualization and study of the bypassed area with a flexible endoscope. ${ }^{1-4,10-12}$

As an increasing number of patients underwent the RYGBP, it became clear that even if they were losing considerable weight, overall results could be improved with somewhat longer limbs. Moreover as more patients with high BMI underwent RYGBP, some authors decided to tailor the limb's length with the BMI, so patients with BMIs $>50$ had longer limbs than those with BMIs $<50$. In patients with BMIs $>50$, the Roux and biliopancreatic limbs were frequently $150 \mathrm{~cm}$ and $100 \mathrm{~cm}$, whereas in those with BMIs $<50$, they were $100 \mathrm{~cm}$ and $50 \mathrm{~cm}$, respectively.

Increasing the limb-lengths also increased the distance between the mouth and the bypassed stomach that currently varies from 200 to $300 \mathrm{~cm}$, which makes the organ difficult to reach with a flexible endoscope.

Many articles have been published regarding the possibility of studying the bypassed stomach. Some authors have suggested inserting a gastrostomy tube with a radiopaque marker to study it endoscopically or radiologically after the surgery, in case of complications such as bleeding or peptic ulcer. ${ }^{13,14}$ On the contrary, Wood et al, ${ }^{15}$ after studying 1,120 patients after RYGBP, concluded that routine gastrostomy tube placement is not necessary in all patients, but only in those at high risk for gastro-enteric obstruction or anastomotic leak.

Silecchia et al $^{16}$ described a minimally invasive approach to the excluded stomach with a multi-slide computed tomography (CT) scan after percutaneous injection of 300-500 mL of saline solution. Upon completion of the procedure, the CT slides were transferred 
as three-dimensional (3D) images. They were able to visualize the bypassed stomach of 5 patients who underwent LRYGBP 9 months before, and concluded that this technique could be considered as the procedure of choice for visualizing the bypassed stomach.

Many patients develop anemia after surgery, and when no cause is detected by examining the upper and lower GI tracts, bleeding from the remnant is suspected and an $\mathrm{H} / \mathrm{K}$-ATPase inhibitor therapy is started. In this study, $80 \%$ of the animals did not show any variation at pathologic examination compared with the control group, based on the concept that serum gastrin levels predict histologic modification of gastric and duodenal mucosae.

Although many retrospective reviews have been reported regarding the long-term physiologic and histologic consequences of LRYGBP on excluded GI segments and hypotheses advanced, few experimental studies have been conducted.

The bypassed stomach has been studied in the past to better understand the changes that RYGBP could create in the bypassed stomach. Printen and Owensby ${ }^{17}$ investigated the vagal innervations of the excluded stomach; they evaluated 25 patients with the Hollander test before and after surgery, and demonstrated that vagal integrity is maintained in the distal pouch and gastric secretory function is basically unchanged following RYGBP.

However, neutralization of gastric acid by ingested food cannot occur in the bypassed segment, and bile reflux may further contribute to mucosal injury. In a study by Sinar et al, ${ }^{11}$ using retrograde duodenogastroscopy to survey both the proximal stomach and the bypassed distal stomach, a significant discrepancy between the severity of endoscopic bile reflux was observed as well as the histologic findings in the remnant stomach. The endoscopic appearance of linear erythema, submucosal hemor- rhage, and superficial erosions described in the distal stomach of postoperative patients did not correlate with the histologic findings. The bile stagnation resulted in slight-to-moderate non-erosive superficial gastritis in $40 \%$ of the patients, and the proximal stomach showed slightly more histologic inflammation than the distal stomach at 1 and 2 years postoperatively. ${ }^{10}$

In our study, serum gastrin levels did not show significant alterations when measured pre-surgery and at 6 months post-surgery (Figure 4). Only one animal had a slight increase of the gastrin from $38 \mathrm{pg} / \mathrm{mL}$ to $40 \mathrm{pg} / \mathrm{mL}$.

Cancer has not been documented as a complication of gastric bypass. The few reports of cancer in the bypassed stomach could represent coincidental findings. On the other hand, gastric cancer in the defunctioned segment of the stomach after RYGBP may be a late event. As the number of patients with a bypassed stomach increase, more cases may conceivably be reported.

Table 2 lists the reports of cancer in the bypassed stomach. These patients developed gastric cancer 5 to 22 years after the original bypass. All had had a Roux-en-Y construction. Contributing factors may include hypochlorhydria, diminished gastrin production, bacterial production, and $H$. pylori infection.

In past animal studies, no change was found in the fasting serum gastrin levels during the 12-month postoperative period, with a marked decrease in group-1 pepsinogen (GIP). Both proximal and distal pouches had a similar increase in basal volume and basal acid output even if a decrease in peak acid output was present. ${ }^{11}$ In another animal model, a significant reduction in serum gastrin levels was found, associated with reduced glandular mucosal weight. ${ }^{12}$

Table 2. Reports of carcinoma in the bypassed stomach after RYGBP

\begin{tabular}{lcccl}
\hline Author & Patients & Age & Years after Surgery & Location \\
\hline Raijman $^{6}(1991)$ & 1 & 38 & 5 & ADC, stomach body \\
Lord $^{7}(1997)$ & 1 & 71 & 13 & 2 ADC, antrum, prepyloric area \\
Khitin $^{5}(2003)$ & 1 & 57 & 22 & ADC, antrum \\
Escalona $^{8}(2005)$ & 1 & 51 & 8 & ADC, antrum \\
\hline
\end{tabular}

$\mathrm{ADC}=$ adenocarcinoma 


\section{Conclusion}

In this study, poor correlation existed between the LRYGBP, fasting serum gastrin levels, and histologic findings. The difference in gastrin levels before and after surgery was minimal and not statistically significant. Moreover, histologic alterations which occurred in only two animals appeared to be unrelated to the physiologic changes that occurred after LRYGBP. This study revealed that LRYGBP is not associated with gastrin and major histologic changes in the bypassed segments at 6 months postoperatively in the porcine model.

We thank Dr. Lydia Petrovic for the review of the histologic slides in this project.

\section{References}

1. Sampalis JS, Liberman M, Auger S et al. The impact of weight reduction surgery on health-care costs in morbidly obese patients. Obes Surg 2004; 14: 939-47.

2. Monteforte MJ, Turkelson CM. Meta-Analysis. Bariatric surgery for morbid obesity. Obes Surg 2000; 10: 391-401.

3. Mason EE, Tang S, Renquist KE et al. A decade of change in obesity surgery. Obes Surg 1997; 7: 189-97.

4. MacLean LD, Rhode BM, Nohr CW. Late outcome of isolated gastric bypass. Ann Surg 2000; 231: 524-8.

5. Khitin L, Roses RE, Birkett DH. Cancer in the gastric remnant after gastric bypass: a case report. Curr Surg 2003; 60: 521-3.

6. Raijman I, Strother SV, Donegan WL. Gastric cancer after gastric bypass for obesity. Case report. J Clin Gastroenterol 1991; 13: 191-4.

7. Lord RV, Edwards PD, Coleman MJ. Gastric cancer in the bypassed segment after operation for morbid obesity. Aust NZ J Surg 1997; 67: 580-2.

8. Escalona A, Guzmán S, Ibàñez L et al. Gastric cancer after Roux-en-Y gastric bypass. Obes Surg 2005; 15 : 423-7.

9. Gentileschi P, Gagner M. Laparoscopic Roux-en-Y gastric bypass for morbid obesity. In: Cueto-Garcia J, Jacobs M, Gagner M, eds. Laparoscopic Surgery. New York: McGraw Hill 2003: 171-8.

10. Park HK, Sinar DR, Sloss RR. Histologic and endoscopic studies before and after gastric bypass. Arch Pathol Lab Med 1986; 110: 1164-7.

11.Sinar DR, Flickinger EG, Harvell JC et al. Physiologic and histologic consequences of gastric bypass surgery in a dog model. Gastroenterology 1984; 86: 1253 (Abst).

12. Young EA, Taylor MM, Taylor MK et al. Gastric stapling for morbid obesity: gastrointestinal response in a rat model. Am J Clin Nutr 1984; 40: 293-302.

13. Fobi MA, Chicola K, Lee H. Access to the bypassed stomach after gastric bypass. Obes Surg 1998; 8: 289-95.

14. Sandbom M, Nyman R, Hedenström $H$ et al. Investigation of the excluded stomach after Roux-enY gastric bypass. Obes Surg 2001; 11: 25-7.

15. Wood MH, Sapala JA, Schuhknecht MP et al. Micropouch gastric bypass: indications for gastrostomy tube placement in the bypassed stomach. Obes Surg 2000; 10: 413-9.

16. Silecchia G, Catalano C, Gentileschi P et al. Virtual gastroduodenoscopy: a new look at the bypassed stomach and duodenum after laparoscopic Roux-en-Y gastric bypass for morbid obesity. Obes Surg 2002; 12: $39-48$.

17. Printen KJ, Owensby M. Vagal innervation of the bypassed stomach following gastric bypass. Surgery 1978; 84: 455-6.

(Received February 1, 2006; accepted April 29, 2006) 